\title{
Spiral instabilities: little interaction with a live halo
}

\author{
J. A. Sellwood, ${ }^{1 \star}$ \\ ${ }^{1}$ Steward Observatory, University of Arizona, $933 \mathrm{~N}$ Cherry Ave, Tucson AZ 85722, USA
}

2 July 2021

\begin{abstract}
In order to address the question of whether spiral disturbances in galaxy discs are gravitationally coupled to the halo, we conduct simulations of idealized models of disc galaxies. We compare growth rates of spiral instabilities in identical mass models in which the halo is held rigid or is represented by particles drawn from an equilibrium distribution function. We examine cases of radial and azimuthal bias in the halo velocity ellipsoid in one of our models, and an isotropic velocity distribution in both. We find at most marginal evidence for an enhanced growth rate of spiral modes caused by a halo supporting response. We also find evidence for very mild dynamical friction between the spiral disturbance and the halo. We offer an explanation to account for the different behaviour between spiral modes and bar modes, since earlier work had found that bar instabilities became significantly more vigorous when a responsive halo was substituted for an equivalent rigid mass distribution. The barely significant differences found here justify the usual simplifying approximation of a rigid halo made in studies of spiral instabilities in galaxies.
\end{abstract}

Key words: galaxies: spiral — galaxies: evolution — galaxies: structure — galaxies: kinematics and dynamics — instabilities

\section{INTRODUCTION}

Theoretical studies of spiral instabilities in galaxies (e.g. Toomre 1981; Bertin et al. 1989; Sellwood \& Kahn 1991) have almost always assumed that bulge and halo components can be treated as inert matter that contributes to the central attraction, but does not otherwise affect the dynamics of the disc component. This assumption is often made in simulations also (e.g. Grand et al. 2012; D'Onghia et al. 2013; Sellwood \& Carlberg 2014; Baba 2015). Exceptionally, Mark (1976) argued that spiral modes would be amplified by the loss of angular momentum to the halo, which he predicted would take place mostly at corotation of the spiral mode. While many simulations of discs embedded in live halos have been performed in subsequent years, there does not appear to have been a quantitative test of Mark's prediction.

It has long been established that strong bars experience dynamical friction from responsive halos (e.g. Sellwood 1980; Weinberg 1985; Debattista \& Sellwood 2000). It has also been found that bar instabilites are more vigorous in responsive halos (e.g. Athanassoula 2002; Saha \& Naab 2013; Berrier \& Sellwood 2016) than in rigid ones, and that the more rapid growth begins at tiny amplitude (Sellwood 2016). This last result suggests that a rigid halo might also be an inadequate approximation for studies of spiral instabilities.

This paper therefore reports a suite of idealized simulations of discs in halos to determine the effect on spiral modes of subsitituting a responsive halo for a rigid one.

\footnotetext{
* E-mail:sellwood@as.arizona.edu
}

\section{TECHNIQUE}

When using simulations to make quantitative measurements of the growth rates of instabilities, it is important to set up the initial model with considerable care in order that it be close to a settled equilibrium. Even quite mild adjustments to the initial model will mask, and perhaps seed, early growth of instabilities, and the instability will be that of the relaxed model and not that of the mass distribution originally intended. Equilibrium models of a disc embedded in a halo present a particular challenge, since few equilibrium distribution functions (DFs) are known for the halo component when a massive disc is present. In this paper we present two separate equilibrium models and provoke a spiral instability in the disc component of each. We then compare the evolution of the instability in both the fully-self consistent models having reponsive halos and cases in which the halos are frozen.

\subsection{Numerical method}

The particles in our simulations move in a $3 \mathrm{D}$ volume that is spanned by two separate grids; a cylindical polar mesh and a much larger spherical grid. The gravitational field is calculated at grid points and interpolated to the position of each particle. The disc particles are initially assigned to the polar grid, while the spherical grid is used for the bulge and halo particles. Naturally, all particles are attracted by all others at every step. A full description of our numerical procedures is given in the on-line manual (Sellwood 2014) and the code itself is available for download.

Tables 1 and 3 give the values of the numerical parameters for most of the simulations presented in this paper. Since the 
Table 1. Numerical parameters for the PK model

Polar grid size

Grid scaling

Vertical spacing

Active sectoral harmonics

Softening length

Spherical grid

Active spherical harmonics

Disc mass fraction

Number of disc particles

Number of halo particles

Basic time-step

Time step zones

gravitational field is a convolution of the mass density with a Green function that is most efficiently computed by Fourier transforms, it is easy to restrict the sectoral harmonics that contribute to the field when using a polar grid. In most simulations we report here, non-axisymmetric forces arising from the particles are confined to just the $m=3$ sectoral harmonic for the PK model ( $\S 3)$ and $m=2$ for the DBH model $(\S 4)$. Aspherical components of forces computed via the spherical grid are similarly confined to the spherical harmonic $l=3$ or 2 .

\subsection{Other details}

We make measurements of the development of nonaxisymmetric disturbances from the azimuthal Fourier coefficients of the mass distribution on the polar grid, generally combining them to report a weighted average of the relative amplitude over a limited radial range. We also compute logarithmic spiral transforms of the disc particle distribution, which is defined for particles of unequal mass as

$$
A(m, \tan \gamma, t)=\frac{\sum_{j=1}^{N} \mu_{j} \exp \left[i m\left(\phi_{j}+\tan \gamma \ln R_{j}\right)\right]}{\sum_{j=1}^{N} \mu_{j}},
$$

where $\left(R_{j}, \phi_{j}\right)$ are the cylindrical polar coordinates of the $j$ th particle of mass $\mu_{j}$ at time $t$, and $\gamma$, the complement to the spiral pitch angle, is the angle between the radius vector and the tangent to an $m$-arm logarithmic spiral, with positive values for trailing spirals.

In order to measure mode frequencies, including growth rates, we fit modes to these data using the procedure described by Sellwood \& Athanassoula (1986). The perturbed surface density of a mode is the real part of

$$
\delta \Sigma(R, \phi, t)=\mathcal{A}_{m}(R) e^{i(m \phi-\omega t)},
$$

where the frequency $\omega=m \Omega_{p}+i \beta$ is complex with $\beta$ being the growth rate. The complex function $\mathcal{A}_{m}(R)$, which is independent of time, describes the radial variation of amplitude and phase of the mode.

Sellwood \& Athanassoula (1986) showed that the mode frequency, especialy the growth rate, could be estimated more precisely from quiet start simulations in which noise was artificially suppressed by placing particles around rings and filtering out the higher sectoral harmonics, allowing a longer period of exponential growth. As this trick is more difficult to accomplish in 3D multi-component models, we here beat down shot noise simply by employing large numbers of particles.

\section{PLUMMER-KUZMIN MODEL}

A composite disc and halo model that is very nearly in equilibrium can be constructed using a Kuzmin disc embedded within a Plummer sphere. The potential of an axisymmetric Kuzmin disc is

$$
\Phi_{\mathrm{K}}(R, z)=-G M\left[R^{2}+(a+|z|)^{2}\right]^{-1 / 2},
$$

(Binney \& Tremaine 2008, eq. 2.68a), where $M$ is the mass of the disc and $a$ is a length scale. That of a Plummer sphere, also of mass $M$ and core radius $a$, is $\Phi_{\mathrm{P}}(r)=-G M\left[r^{2}+\right.$ $\left.a^{2}\right]^{-1 / 2}$, which is identical in the $z=0$ plane and differs only slightly for $|z|>0$. Thus, a composite model of two superposed concentric components having equal length scales $a$, with disc mass $f_{d} M$ and sphere mass $\left(1-f_{d}\right) M$, will have the same potential in the mid-plane. We describe this as the PK model, and it is natural to adopt units such that $G=$ $M=a=1$. Though hardly a realistic galaxy model, it has the following advantages for our study.

Kalnajs (1976) gave a family of equilibrium DFs having a parameter $m_{K}$ that determines the degree of random motion in the razor-thin Kuzmin disc model. Also Dejonghe (1987) derived a family of DFs having a parameter $q$ that determines the shape of the velocity ellipsoid in the Plummer sphere. Neither of these DFs would yield perfect equilibrium in a composite model, however. The Plummer sphere would be affected by the slight difference of the disc contribution to the potential away from the mid-plane, which we find is small enough to ignore. More significantly, the disc needs to be thickened to avoid local disc instabilities. A disc lacking random motion is Jeans unstable (Toomre 1964), while a razor-thin disc having a finite radial velocity dispersion suffers from buckling instabilities (Toomre 1966; Araki 1985). Since thickening the disc weakens the central attraction in the mid-plane, as does gravity softening, radial balance requires a compensating additional central force that is the difference between that expected from the total mid-plane potential and the numerically determined attraction from the particles in the simulation. The radial variation of the extra central force needed in the mid-plane is computed at the start, and is applied as a spherically symmetric term that is held fixed throughout the simulation. It can be thought of as an additional rigid halo, although the implied mass is just a few percent of that of the disc.

The principal advantage of pursuing these oversimplified galaxy models is that we can employ anisotropic DFs of the Plummer sphere in our experiments. We wish to determine the influence of a responsive halo on the spiral instabilities within the disc, and this model enables us to vary the shape of halo velocity ellipsoid from radial bais, through isotropy to azimuthal bias, as was also exploited by Sellwood (2016) in his study of bar instabilities. Furthermore, we are able to set up good equilibria for both the disc and halo by drawing particles from the known DFs, using the method described by Debattista \& Sellwood (2000). We use the 1D Jeans equation to set up the vertical balance of the disc particles, which yields an acceptable equilibrium when the radial velocity dispersion is modest. The resulting model is very close to overall equilibrium, and the mass profiles of both disc and halo remain indistinguishable from the initial set up during the entire linear growth phase of the instability.

Since the bar instability in a disc is made even more vigor- 
Table 2. Mode frequencies fitted to the PK models

\begin{tabular}{lcc} 
Halo & $m \Omega_{p}$ & $\beta$ \\
\hline Rigid & $0.663 \pm 0.002$ & $0.039 \pm 0.004$ \\
Isotropic & $0.659 \pm 0.001$ & $0.040 \pm 0.002$ \\
Azimuthal bias & $0.653 \pm 0.002$ & $0.045 \pm 0.004$ \\
Radial bias & $0.660 \pm 0.001$ & $0.041 \pm 0.002$
\end{tabular}

ous by a live halo, spiral instabilities would be overwhelmed by the bar mode unless the disc mass fraction were very low, and then the spirals would be weak and multi-armed. We therefore restrict non-axisymmetric disturbance forces in these simulations to the $m=3$ sectoral harmonic in order to inhibit the bar mode, while allowing a single spiral mode.

We provoke a spiral instability at a predictable radius by creating a groove in the angular momentum profile of the disc (Sellwood \& Kahn 1991). In this case, we multiply the DF given by Kalnajs (1976), which is a function of the two classical integrals $E$ and $L_{z}$, by the Lorentzian factor

$$
\left[1-\frac{D w_{j}^{2}}{\left(L_{z}-L_{z}^{*}\right)^{2}+w_{j}^{2}}\right],
$$

and select equal mass disc particles from this modified DF. In expression (4), $D$ is the relative depth of the groove, $w_{j}$ is the width parameter, and $L_{z}^{*}$ is the angular momentum of the groove centre. Note that this is a groove in the distribution of guiding centres and epicyclic blurring implies a shallower and broader dent in the surface density profile.

For our simulations, we choose $f_{d}=1 / 3$ and the disc DF parameter $m_{K}=30$, which yields $Q \simeq 2$ in the disc centre and decreasing gently to $Q \simeq 1.2$ in the outer disc. The groove parameters are: $L_{z}^{*}=1.4, w_{j}=0.04$ in our adopted units, and $D=0.4$. The halo DF parameters in the three models with live halos are $q=0$ for an isotropic velocity distribution, $q=2$ for maximum radial bias, and $q=-15$ for a strong azimuthal bias. Note that in all cases the central parts of the halo are closely isotropic, while anisotropies increase with radius beyond the core.

\subsection{Results}

We have conducted four simulations of the PK model, each employing $100 \mathrm{M}$ particles in the disc component. They differ in the shape of velocity ellipsoid in the live halo component, which also has $100 \mathrm{M}$ particles and in one case the halo was replaced by a rigid unresponsive mass of the same density. The groove provoked an $m=3$ spiral instability in all four cases. The power spectrum in the middle panel of Figure 1 indicates that the isotropic halo model supported a single dominant mode with corotation at $R \simeq 2.6$; power spectra from the other three simulations were very similar. The upper panel shows the growth of the $m=3$ disturbance amplitude in the disc component in each PK model. The instability took slightly different lengths of time to emerge from the noise, but over the interval $100 \leq t \leq 180$ the slope of each line in this log-linear plot, which is the growth rate of the mode, is remarkably similar. This finding is already in strong contrast with the behaviour reported for bar instabilities by Sellwood (2016), where growth rates in models with different halo velocity ellipsoids were between 2 and 5 times greater than the bar mode in a disc with rigid halo.

We have estimated the mode frequencies in all four models
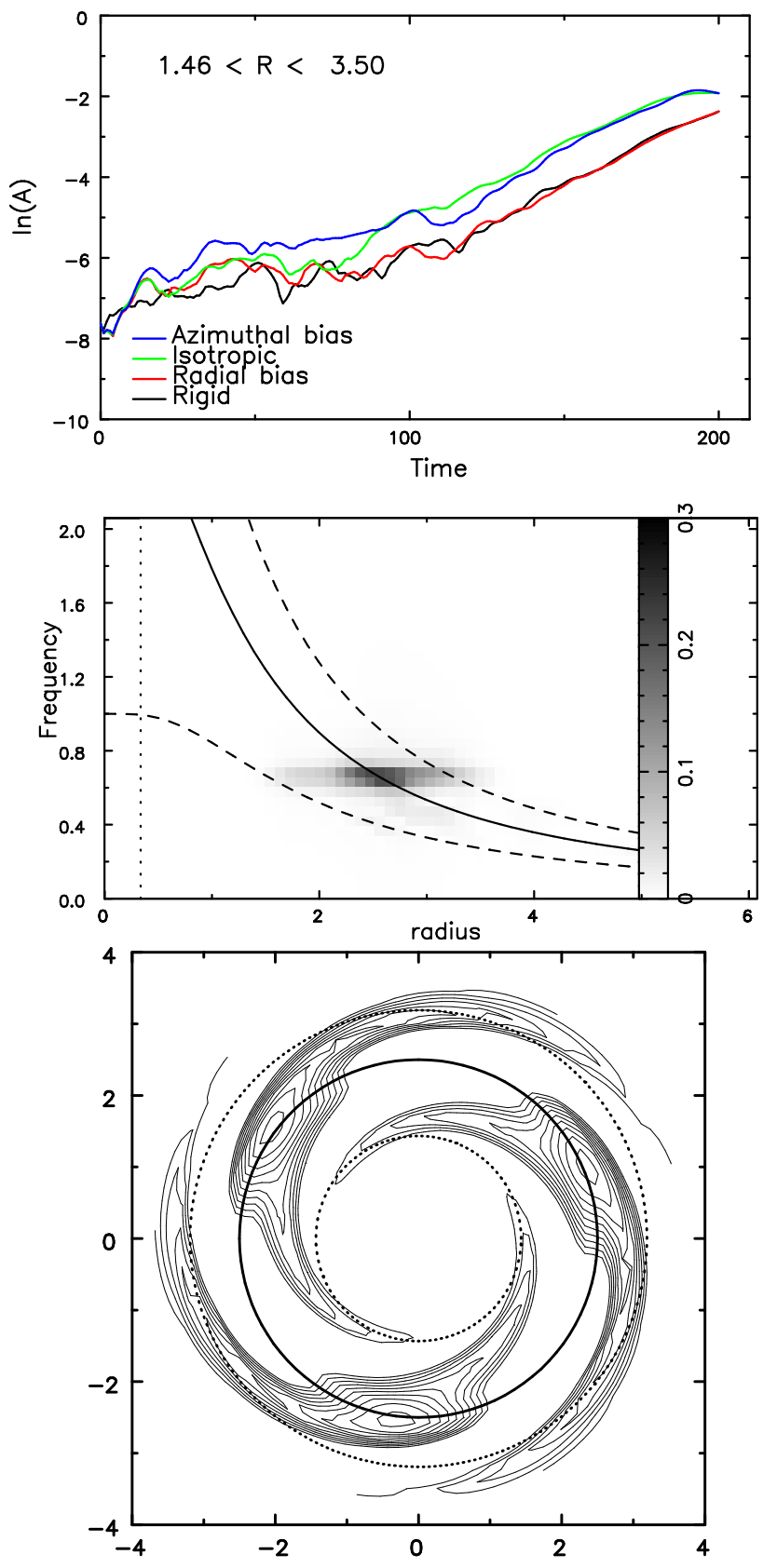

Figure 1. Top panel: The weighted average of the $m=3$ relative overdensity in the indicated radial range over which the spiral mode is strongest. The four separate simulations differ only in the halo DF as indicated by the line colours, and the black line is for a rigid halo. Middle panel: Power spectrum of the $m=3$ component from the isotropic halo model over the time interval $61 \leq t \leq 180$ indicating a single dominant mode. The solid curve marks $m \Omega_{c}$ and the dashed curves $m \Omega_{c} \pm \kappa$. Bottom panel: The mode fitted to the data from the isotropic halo model over the time interval $80 \leq t \leq 180$. The corotation radius is marked by the full-drawn circle and the Lindblad resonances by the dotted circles.

by the method described in Sellwood \& Athanassoula (1986), fitting eq. (2) to data from the simulations over selected time ranges. We fit both the disturbance density and logarithmic spiral transforms, selecting differing time ranges, radial ranges, and numbers of values of $\tan \gamma$. We use these differ- 


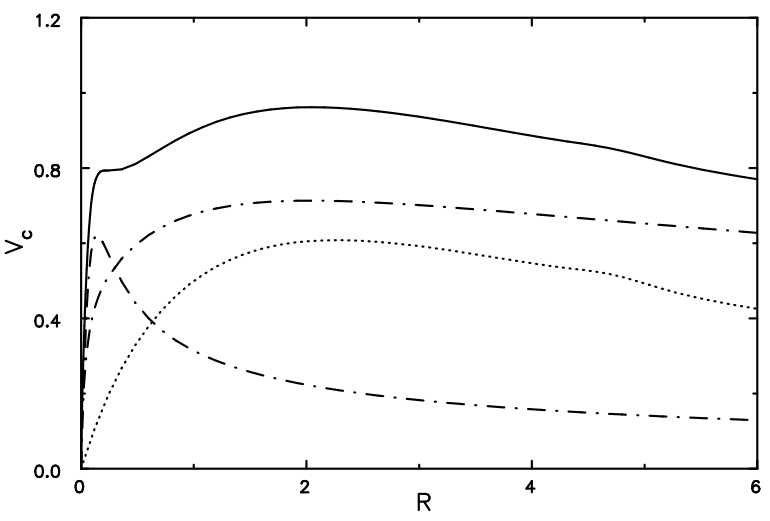

Figure 2. The initial rotation curve of the disc-bulge-halo model (solid curve) measured from the particles. The disc contribution is given by the dotted line, while the dot-dash lines indicate those of the bulge and compressed halo.

ent fits to obtain estimates of the uncertainties in the fitted frequencies. The best fit values are presented in Table 2, with the quoted uncertainties bracketing the full range of credible fitted values in each case. The mode shape drawn in bottom panel of Figure 1 is the real part of best fit function $\mathcal{A}_{m}(R)$ (eq. 2), and the contours are of only the positive relative overdensity; the principal resonances are marked by circles.

Only the model with the strong azimuthal bias has a higher growth rate than the others, though barely so. This finding is supported by the blue line in the upper panel of Figure 1, which may have a visibly steeper slope over the time interval $100<t<180$ than the other three cases. But it seems that the mode grows at essentially the same rate in the rigid halo as in the isotropic and radially biased halos, and scarcely any faster in the azimuthally biased halo.

A supporting response from the halo would most likely come from those orbits that are near circular, directly rotating, and oriented close to the disc plane, since they would effectively augment the disc, as Sellwood (2016) argued for bar modes. As the number of such orbits should be greatest when the halo velocity ellipsoid has a strong azimuthal bias, a significant supporting response seems physically reasonable, but even in this case the growth rate is enhanced by no more than $\sim 10 \%$. However, the trefoil spiral in these models may not induce as strong a halo supporting response as would a bi-symmetric spiral, as has been established for bar modes.

\section{DISC-BULGE-HALO MODEL}

In order to study the behaviour in a somewhat more realistic model, and to extend it to bisymmetric spirals, we adopt an exponential disc model embedded within a halo and a central bulge. The bulge is required both to create the steeper inner rise in the rotation curve typical of a large spiral galaxy, and also to prevent bar instabilities (Toomre 1981) while allowing $m=2$ spirals.

- The exponential disc has the surface density

$$
\Sigma(R)=\frac{M_{d}}{2 \pi R_{d}^{2}} \exp \left(-R / R_{d}\right)
$$

which we taper to zero over the radial range $4.5<R / R_{d}<5$,

Table 3. Numerical parameters for DBH model

Polar grid size

Grid scaling

Vertical spacing

Active sectoral harmonics

Softening length

Spherical grid

Active spherical harmonics

Number of disc particles

Number of halo particles

Number of bulge particles

Basic time-step

Time step zones

$$
\begin{aligned}
& 85 \times 128 \times 125 \\
& R_{d}=10 \text { grid units } \\
& \delta z=0.02 R_{d} \\
& m=0,2 \\
& R_{d} / 10 \\
& 501 \text { shells } \\
& l=0,2 \\
& 10^{8} \\
& 10^{8} \\
& 10^{7} \\
& \left(R_{d}^{3} / G M\right)^{1 / 2} / 320
\end{aligned}
$$

and $M_{d}$ is the mass of the notional infinite disc. The vertical density profile of the disc is Gaussian with a scale $0.1 R_{d}$.

- We use a dense Plummer sphere for the bulge, which has a mass of $0.1 M_{d}$ and core radius $a=0.1 R_{d}$, and adopt the isotropic DF given by Dejonghe (1987). The bulge is dense enough that it dominates the central attraction in the inner part of our galaxy model, and the analytic DF is close to equilibrium despite the presence of the disc and halo.

- We employ a Hernquist (1990) model for the halo that has the density profile

$$
\rho(r)=\frac{M_{h} b}{2 \pi r(b+r)^{3}},
$$

where $M_{h}$ is the total mass integrated to infinity, and $b$ is a length scale. We choose $M_{h}=5 M_{d}$ and $b=4 R_{d}$. Naturally, the isotropic DF that Hernquist derived for this isolated mass distribution would not be in equilibrium when the disc and bulge are added, so we use the adiabatic compression procedure described by Sellwood \& McGaugh (2005) that starts from the isotropic DF given by Hernquist and uses the invariance of both the radial and azimuthal actions to compute a revised density profile and DF as extra mass is inserted. The revised DF has a slight radial bias. We also apply an outer cutoff to the selected particles that excludes any with enough energy ever to reach $r>10 b$, which causes the halo density to taper smoothly to zero at that radius.

The resulting rotation curve of this model is illustrated in Figure 2, where we have adopted units such that $G=$ $M_{d}=R_{d}=1$. We also adopt a constant value of $Q=1.5$ at all radii to determine the radial velocity dispersion of the disc particles. Although the disc is quite massive, the high value of the epicyclic frequency, $\kappa$, near the centre in particular implies disc random velocities are modest everywhere, and the Jeans equations in the epicyclic approximation (Binney \& Tremaine 2008) yield an excellent equilibrium.

Again we wish to seed a spiral instability at a predictable radius, but require a different strategy in this case because we do not select disc particles from a DF. Instead we create a groove in the disc surface density profile by multiplying the mass of every disc particle by the same expression as above (eq. 4). As before, this sharp groove in angular momentum is blurred by epicycle motions.

We run three cases placing the groove centre at $L_{z}^{*}=1.5$, 2 , and 2.5. In each case we choose, $w_{j}=0.04$ in our adopted units, and $D=0.6$, and run both live and rigid halos and bulges. We employ $100 \mathrm{M}$ particles in each of the disc and 

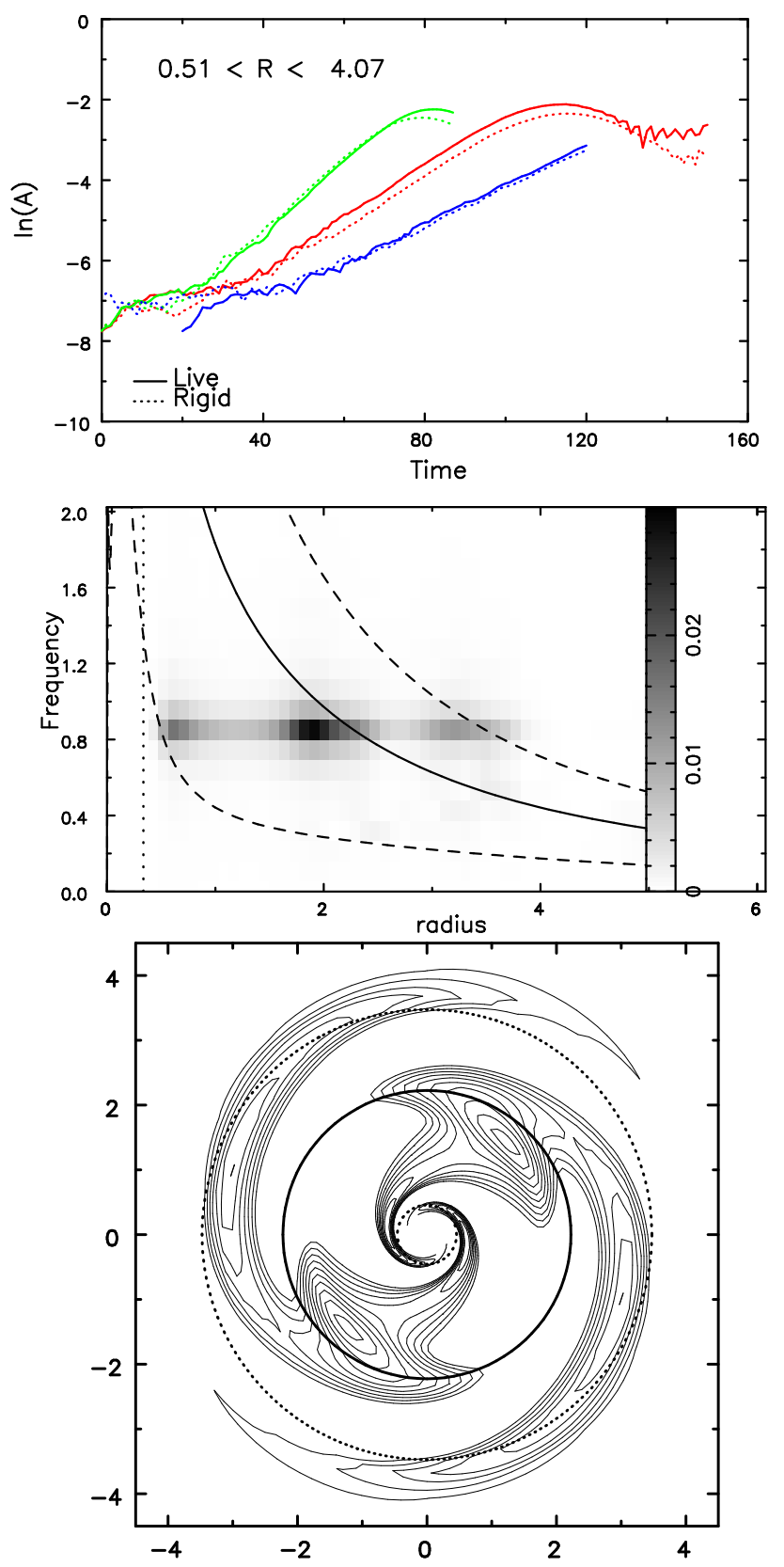

Figure 3. Top panel: The weighted average of the $m=2$ relative overdensity in the indicated radial range over which the spiral mode is strongest. The six simulations of this DBH model have rigid (dotted lines) and live halos (solid lines) and $L_{z}^{*}=2$ (red), $L_{z}^{*}=1.5$ (green), and $L_{z}^{*}=2.5$ (blue). The blue lines have been shifted horizontally to show the similarity of slopes without overlapping with the others. Middle panel: Power spectrum of the $m=3$ component from the live halo simulation with $L_{z}^{*}=2$ over the time interval $30 \leq t \leq 80$ indicating a single dominant mode. Bottom panel: The mode fitted to the data from the live halo model over the time interval $30 \leq t \leq 90$. The lines in the middle panel and the circles in the bottom panel have the same meanings as in Fig. 1.
Table 4. Mode frequencies fitted to the DBH models

\begin{tabular}{lccc} 
Halo & $L_{z}^{*}$ & $m \Omega_{p}$ & $\beta$ \\
\hline Rigid & 1.5 & $1.109 \pm 0.001$ & $0.093 \pm 0.001$ \\
Live & 1.5 & $1.108 \pm 0.004$ & $0.095 \pm 0.001$ \\
Rigid & 2 & $0.855 \pm 0.001$ & $0.065 \pm 0.002$ \\
Live & 2 & $0.841 \pm 0.001$ & $0.069 \pm 0.003$ \\
Rigid & 2.5 & $0.674 \pm 0.001$ & $0.049 \pm 0.001$ \\
Live & 2.5 & $0.667 \pm 0.003$ & $0.051 \pm 0.001$
\end{tabular}

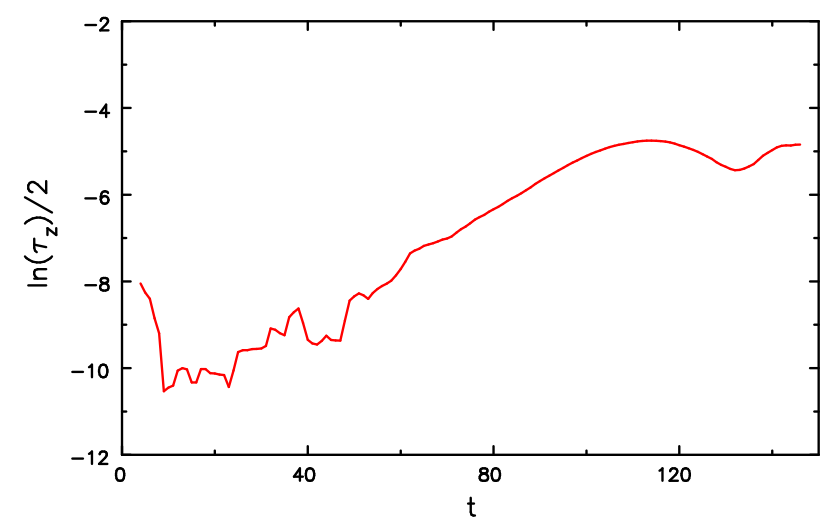

Figure 4. The time evolution of the square-root of the torque between the disc and halo for the $L_{z}^{*}=2$ case. Notice the quantitative scaling similarity between the solid red line in the top panel of Fig. 3 and that in this figure, indicating that the torque varies as the square of the mode amplitude.

halo and $10 \mathrm{M}$ in the bulge. The other numerical parameters are given in Table 3.

\subsection{Results}

The middle panel of Figure 3 gives the power spectrum in the model with the $L_{z}^{*}=2$ and live halo while the upper panel indicates the growth of the $m=2$ disturbance in all six simulations with this galaxy model; those having a responsive halo and bulge and marked with solid lines and those for which both spherical components are rigid have dotted lines. As for the PK models, differences between the live and rigid halos for all three diferent groove radii are tiny - the bisymmetric spiral mode appears to grow at roughly the same rate in the live as in the rigid halo in each case. Naturally, the mode pattern speed and growth rate decrease as the groove radius is increased because the dynamical clock runs more slowly. The fitted frequencies in the six runs are listed in Table 4, from which it may be seen that the fitted growth rates in the live halo runs are fractionally higher than in the rigid halo cases, but the differences are all within the estimated uncertainties.

The mode shape for the $L_{z}^{*}=2$ case is illustrated in the bottom panel of Figure 3. The pronounced kink across corotation reflects the mechanism of the groove mode in a cool disc (Sellwood \& Kahn 1991). The mode shape is very similar for the rigid halo case and, apart from a different spatial scale, for the other two groove radii.

The live halo causes other differences, apart from a marginal increase in growth rate. For example in the $L_{z}^{*}=2$ case, the saturation amplitude of the mode is some $20 \%$ higher in the live halo than in the rigid. Also a small frac- 
tion, $\sim 0.2 \%$, of the disc angular momentum is taken up by the halo, and a tiny fraction, $\sim 0.004 \%$, by the bulge. Though always small, the torque on the halo (Figure 4) increases approximately as the square of the mode amplitude, i.e. at twice the growth rate, until it saturates, which is the scaling expected in dynamical friction as a perturber is decelerated by its wake (Tremaine \& Weinberg 1984; Sellwood 2006; Binney \& Tremaine 2008).

It is noteworthy that we could not find any evidence for a bar instability in this model, in agreement with the linear theory prediction for a model having a dense centre (Toomre 1981). However, inner Lindblad resonance damping of disturbances is a prediction of small amplitude perturbation theory and the strong bisymmetric spiral extends over a broad radial range, yet it still did not lead to non-linear trapping into a bar in the inner disc, at least for the duration of the simulations.

\section{DISCUSSION}

It may seem strange that bar instabilities in responsive halos grow several times faster than in the equivalent rigid halo (Athanassoula 2002; Sellwood 2016), while a live halo has a tiny effect on even bisymmetric spiral modes. Note that this difference appears in the linear growth phase of the instabilities and therefore the fact that bar modes may ultimately rise to larger amplitude than do spirals cannot account for this particular discrepancy.

Another significant difference between the two cases is the second order dependence of the torque from the spiral instability on the halo, which contrasts with the first order torque reported by Sellwood (2016, his fig. 2) for the strong halo enhancement of bar instabilities.

The self-consistency requirement of a linear instability is that the disturbance density results from orbit deflections caused by the disturbance potential. Thus, when a live halo has a large effect on the growth rate of the bar mode, its supporting response must be an integral part of the instability of the disc-plus-halo system. Furthermore, the linear growth of the disc-halo torque indicates that the instability is driven, in part at least, by angular momentum loss from the disc.

In contrast, we report here that a live halo has little effect on spiral modes, indicating that the halo takes no significant part in this type of instability in the disc-halo system. Dynamical friction on the disc disturbance as it moves through the non-rotating halo is inevitable, but is weak and second order.

So why do we find a negligible halo response to spiral modes, but had previously reported an enthusiastic supporting response to bar modes? We suggest the following reason: Lynden-Bell (1979) demonstrated that orbits of arbitrary eccentricity that are subject to the gravitational influence of a weak bar generally will be repelled from alignment with the bar except in the inner parts of galaxy models having a quasi-uniform core, i.e. where the rotation curve rises roughly linearly. The bar unstable models simulated by both Athanassoula (2002) and by Sellwood (2016) had slowly rising rotation curves, and therefore the prograde halo orbits near the disc plane in the inner parts of those models would be attracted into co-alignment with the growing bar perturbation in the disc, creating a strong supporting response from the halo that enhances the growth rate. Where the rotation curve is flat or declining, the response of higher angular momentum halo particles does not reinforce the perturbation, and disturbances in the disc are little affected by a responsive halo. Thus an aligning halo response for the bisymmetric spiral in the DBH model would not be expected because the central bulge eliminates an extensive region where the potential is quasi-harmonic. We have reworked Lynden-Bell's analysis for the trefoil spiral in the PK model, finding that the key gradient $\partial \Omega_{i} /\left.\partial L_{z}\right|_{J_{f}}$ indicates anti-alignment in the region of corotation for the groove mode, and therefore no supporting response from halo orbits of any eccentricity. Here $\Omega_{i}=\Omega_{\phi}-\Omega_{R} / 3$ and the fast action $J_{f}=L_{z} / 3+J_{R}$; see Lynden-Bell (1979) for a fuller explanation. This argument would seem to account both for previously published results for the bar mode and for the contrasting finding for spiral modes in this paper.

\section{CONCLUSIONS}

We have constructed two equilibrium models of moderately heavy discs embedded in live halos. We created grooves in both discs in order to provoke strong spiral instabilities and have compared the growth rates of the spiral modes in simulations that employed both rigid and live halos. We cannot exclude that the spiral growth rate was increased by the halo response in both models; there was a hint of a higher growth rate for the $m=2$ spiral mode for all three grooves in the live halo case in the DBH model and a slightly more significant enhanced growth rate for the $m=3$ mode in the PK model when the halo velocity ellipsoid was strongly azimuthally biased, but the growth rate differences were small. Since halos in real galaxies are not expected to have azimuthally biased velocity ellipsoids, this mild boost to spiral growth rates, if real, is unlikely to be of relevance to the development of spirals in galaxies.

We did find evidence for additional differences between the live and rigid halos in the DBH models. The halo was weakly torqued by dynamical friction from the spiral mode, but the loss of angular momentum from the disc was only $0.2 \%$ by the end of the simulation and much less during the linear growth phase. This exchange may have allowed the final spiral amplitude to be about $20 \%$ greater in the live halo case.

The results reported here justify the usual assumption made in theoretical work and in simulations that a frozen halo is an adequate approximation when considering the dynamics of spiral formation.

\section{ACKNOWLEDGEMENTS}

The author thanks Scott Tremaine for some very helpful comments on a draft of this paper, Ray Carlberg for useful correspondence, and an anonymous referee for a careful reading of the paper. JAS acknowledges the continuing hospitality of Steward Observatory.

\section{DATA AVAILABILITY}

The data from the simulations reported here can be made available on request. The simulation code can be downloaded from http://www.physics.rutgers.edu/galaxy 


\section{REFERENCES}

Araki, S. 1985, PhD thesis., MIT.

Athanassoula, E. 2002, ApJ, 569, L83

Baba, J. 2015, MNRAS, 454, 2954

Berrier, J. \& Sellwood, J. A. 2016, ApJ, 831, 65

Bertin, G., Lin, C. C., Lowe, S. A. \& Thurstans, R. P. 1989, ApJ, 338, 104

Binney J. \& Tremaine S. 2008, Galactic Dynamics 2nd ed. (Princeton University Press, Princeton NJ)

Debattista, V. P. \& Sellwood, J. A. 2000, ApJ, 543, 704

Dejonghe, H. 1987, MNRAS, 224, 13

D'Onghia, E., Vogelsberger, M. \& Hernquist, L. 2013, ApJ, 766, 34

Grand, R. J. J., Kawata, D. \& Cropper, M. 2012, MNRAS, 421, 1529

Hernquist, L. 1990, ApJ, 356, 359

Kalnajs, A. J. 1976, ApJ, 205, 751

Lynden-Bell, D. 1979, MNRAS, 187, 101

Mark, J. W-K. 1976, ApJ, 206, 418

Saha, K. \& Naab, T. 2013, MNRAS, 434, 1287

Sellwood, J. A. 1980, A\&A, 89, 296

Sellwood, J. A. 2006, ApJ, 637, 567

Sellwood, J. A. 2014, arXiv:1406.6606 (on-line manual: http://www . physics.rutgers.edu/ sellwood/manual.pdf)

Sellwood, J. A. 2016, ApJ, 819, 92

Sellwood, J. A. \& Athanassoula, E. 1986, MNRAS, 221, 195

Sellwood, J. A. \& Carlberg, R. G. 2014, ApJ, 785, 137

Sellwood, J. A. \& Kahn, F. D. 1991, MNRAS, 250, 278

Sellwood, J. A. \& McGaugh, S. S. 2005, ApJ, 634, 70

Toomre, A. 1964, ApJ, 139, 1217

Toomre, A. 1966, in Geophysical Fluid Dynamics, notes on the 1966 Summer Study Program at the Woods Hole Oceanographic Institution, ref. no. 66-46, p 111

Toomre, A. 1981, In "The Structure and Evolution of Normal Galaxies", eds. S. M. Fall \& D. Lynden-Bell (Cambridge, Cambridge Univ. Press) p. 111

Tremaine, S. \& Weinberg, M. D. 1984, MNRAS, 209, 729

Weinberg, M. D. 1985, MNRAS, 213, 451

This paper has been typeset from a $\mathrm{T}_{\mathrm{E}} \mathrm{X} / \mathrm{LAT} \mathrm{T} \mathrm{X}$ file prepared by the author. 